\title{
Konvensional Realisasi Dari Fungsi Transfer Dalam Bentuk Kanonik Terkontrol
}

\author{
(Realization of Transfer Functions in a Controlled Canonical Form)
}

\author{
Nurweni Putri ${ }^{1}$, Muhafzan ${ }^{2}$ \\ ${ }^{I}$ Dosen Prodi Matematika Universitas Dharma Andalas ; Co-author : nurweniputri@gmail.com \\ ${ }^{2}$ Dosen Jurusan Matematika FMIPA Universitas Andalas
}

\begin{abstract}
Abstrak: Sistem kontrol merupakan suatu alat untuk mengendalikan dan mengatur keadaan dari suatu sistem. Dalam teori kontrol, suatu sistem dapat direpresentasikan dengan beberapa cara yang berbeda. Dalam penelitian ini akan dibahas cara menentukan representasi ruang keadaan dari fungsi transfer dalam bentuk kanonik terkontrol untuk sistem SISO dan MIMO. Dalam literatur, permasalahan ini dikenal dengan realisasi. Masalah ini ekivalen dengan bagaimanakah cara menentukan matriks A, B, $\mathrm{C}$ dan D sedemikan sehingga:

$$
H(s)=C(s I-A)^{-1} B+D,
$$
\end{abstract}

Dimana matriks A, B, C dan D tersebut dalam bentuk kanonik terkontrol

Kata kunci: Fungsi transfer, realisasi, kanonik terkontrol

\begin{abstract}
Control system is a tool to control and regulate the state of a system. In control theory, a system can be represented in several different ways. In this study we discuss how to determine state space representations of transfer functions in controlled canonical forms for SISO and MIMO system. In the literature, this problem is known as realization. This problem is equivalent to how to determine the matriks A, B, C, and D so that

$$
H(s)=C(s I-A)^{-1} B+D,
$$
\end{abstract}

where matriks $\mathrm{A}, \mathrm{B}, \mathrm{C}$, and $\mathrm{D}$ are in a controlled canonical form.

Keywords: Transfer function,realization, controlled canonical.

\section{PENDAHULUAN}

Diberikan suatu sistem kontrol

$$
\begin{aligned}
& \dot{\boldsymbol{x}}(t)=A \boldsymbol{x}(t)+B \boldsymbol{u}(t) \\
& \dot{\boldsymbol{x}}(t)=A \boldsymbol{x}(t)+B \boldsymbol{u}(t)
\end{aligned}
$$

dimana $\boldsymbol{x}(t) \in \mathbb{R}^{n}, \boldsymbol{u}(t) \in \mathbb{R}^{m}, \boldsymbol{y}(t) \in \mathbb{R}^{p}$, $A \in \mathbb{R}^{n \times m}, \mathrm{~B} \in \mathbb{R}^{n \times m}, C \in \mathbb{R}^{p \times m}, D \in$ $\mathbb{R}^{p \times m}$.

Dalam hal ini, $\boldsymbol{x}(t)$ menyatakan variabel keadaan, $\boldsymbol{u}$ menyatakan variabel kontrol (input), $\boldsymbol{y}$ menyatakan output dan $t$ menyatakan waktu (Ogata, 2002).

Fungsi transfer untuk sistem (0.1) didefinisikan sebagai perbandingan transformasi Laplace output terhadap transformasi Laplace input dengan asumsi semua kondisi awal sama dengan nol dan dinotasikan dengan $H(s)$ yaitu

$$
H(s)=\frac{Y(s)}{U(s)}
$$

dimana $Y(s)$ adalah transformasi Laplace dari $\boldsymbol{y}(t)$ dan $U(s)$ adalah transformasi Laplace dari $\boldsymbol{u}(t)$.

Dari denisi ini jelas bahwa jika diberikan suatu sistem kontrol linier, maka fungsi transfernya sebaliknya, jika diberikan suatu fungsi transfer dengan mudah dapat ditentukan. Namun $H(s)$, bagaimanakah bentuk dari sistem kontrol liniernya. Dalam literatur, masalah ini dikenal sebagai masalah realisasi. Masalah ini juga ekivalen dengan bagaimanakah bentuk matriks $A, B$, $C$ dan $D$ dari suatu fungsi transfer $H(s)$ yang diberikan sedemikian sehingga

$$
H(s)=C(s I-A)^{-1} B+D,
$$

Pada penelitian ini akan dikaji permasalahan realisasi dari fungsi transfer dalam bentuk kanonik terkontrol, yaitu jika diberikan suatu fungsi transfer, maka bagaimanakah bentuk representasi ruang 
keadaan yang berkaitan dengan matriks $A$, $B, C$ dan $D$ dimana matriks-matriks tersebut

\section{TINJAUAN PUSTAKA}

Dalam teori kontrol, fungsi transfer (fungsi alih) biasanya digunakan untuk mengkarakteristikkan hubungan antara komponen input output yang dapat diberikan oleh persamaan diferensial linear invarian waktu. Fungsi transfer dari suatu persamaan diferensial linier invariant waktu didenisikan sebagai perbandingan antara transformasi Laplace dari output (fungsi respon) dengan transformasi Laplace dari input dengan asumsi bahwa syarat awal adalah nol. Suatu persamaan diferensial linier invariant waktu

$$
\begin{gathered}
a_{n} y^{(n)}+a_{n-1} y^{(n-1)}+\cdots+a_{1} y+a_{0}= \\
b_{m} u^{(m)}+b_{m-1} u^{(m-1)}+\cdots+b_{0}
\end{gathered}
$$

Dimana $\quad n \geq m, y^{n}=\frac{d^{m} y}{d t^{n}}, u^{m}=\frac{d^{m} u}{d t^{m}}, \quad y$ adalah output dan $\boldsymbol{u}$ adalah input (Ogata, 2002).

Sistem ini dapat diubah menjadi sistem persamaan diferensial linier orde satu dengan cara mendenisikan $n$ variabel baru. Misalkan

$$
\begin{aligned}
& x_{1}=y-\beta_{0} u \\
& x_{2}=\dot{y}-\beta_{0} \dot{u}-\beta_{1} u=\dot{x}_{1}-\beta_{1} u \\
& x_{3}=\ddot{y}-\beta_{0} \ddot{u}-\beta_{1} \dot{u}-\beta_{2} u=\dot{x}_{2}-\beta_{2} u \text { (1.2) } \\
& \quad \vdots \\
& x_{n}=y^{(n-1)}-\beta_{0} u^{(n-1)}-\beta_{1} u^{(n-2)}-\cdots \beta_{n-2}-\beta_{n-1} u \\
& =\dot{x}_{n-1}-\beta_{n-1} u
\end{aligned}
$$

dimana $\beta_{0}, \beta_{1}, \ldots, \beta_{n}$ ditentukan dari

$\beta_{0}=b_{0}$

$\beta_{1}=b_{1}-a_{1} \beta_{0}$

$\beta_{2}=b_{2}-a_{1} \beta_{1}-a_{2} \beta_{0}$

$\beta_{3}=b_{3}-a_{1} \beta_{2}-a_{2} \beta_{1}-a_{3} \beta_{0}$

$\beta_{n}=b_{n}-a_{1} \beta_{n-1}-\cdots-a_{n-1} \beta_{1}-a_{n} \beta_{0}$

Dengan pemilihan variabel keadaan ini, diperoleh

$$
\begin{aligned}
& \dot{x}_{1}=x_{2}+\beta_{1} u \\
& \dot{x}_{2}=x_{3}+\beta_{2} u
\end{aligned}
$$

dalam bentuk kanonik terkontrol.

$$
\begin{aligned}
& \dot{x}_{n-1}=c u \\
& \dot{x}_{n}=-a_{n} x_{1}-a_{n-1} x_{2}-\cdots-a_{1} x_{n}-\beta_{n} u
\end{aligned}
$$

Dalam bentuk persamaan keadaan dan persamaan output, persamaan terakhir dapat ditulis menjadi (0.1), dimana

$$
\begin{gathered}
A=\left[\begin{array}{cccc}
0 & 1 & \cdots & 0 \\
0 & 0 & \cdots & 0 \\
\vdots & \vdots & \ddots & 1 \\
-a_{n} & -a_{n-1} & \cdots & -a_{1}
\end{array}\right], \quad B=\left[\begin{array}{c}
\beta_{1} \\
\beta_{2} \\
\vdots \\
\beta_{n}
\end{array}\right] \\
C=\left[\begin{array}{llll}
1 & 0 & \cdots & 0
\end{array}\right], \quad D=\beta_{0}=b_{0}
\end{gathered}
$$

Dengan asumsi bahwa semua syarat awal bernilai nol, maka fungsi transfer dari (1.1) adalah

$$
\begin{aligned}
G(s) & =\frac{\mathcal{L}[\text { Output }]}{\mathcal{L}[\text { Input }]}=\frac{Y(s)}{U(s)} \\
& =\frac{b_{m} s^{m}+b_{m-1} s^{m-1}+\cdots+b_{1} s+b_{0}}{a_{n} s^{n}+a_{n-1} s^{n-1}+\cdots+a_{1} s+a_{0}} .
\end{aligned}
$$

Untuk sistem MIMO, fungsi transfer G(s) didefinisikan sebagai

dimana

$$
\boldsymbol{Y}(s)=G(s) \boldsymbol{U}(s)
$$

$$
Y(s)=\left[\begin{array}{c}
y_{1}(s) \\
y_{2}(s) \\
\vdots \\
y_{p}(s)
\end{array}\right], \quad U(s)=\left[\begin{array}{c}
u_{1}(s) \\
u_{2}(s) \\
\vdots \\
u_{p}(s)
\end{array}\right]
$$

Secara umum, suatu matriks fungsi transfer $G(s)$ berukuran $p \quad x \quad m$ direpresentasikan sebagai,

dimana

$$
G(s)=\frac{N(s)}{d(s)}
$$

$$
d(s)=s^{n}+a_{n} s^{n-1}+\cdots+a_{1} s+a_{0}
$$

dan

$$
N(s)=N_{n-1} s^{n-1}+N_{n-2} s^{n-2}+\cdots+N_{1} s+N_{0}
$$


Elemen dari matriks $N(s)$ adalah polinomial dalam $s$ dan $N_{0}, N_{l}, \ldots, N_{n-1}$ adalah matriks berukuran $p \quad x \quad m$ serta $d(s)$ merupakan perseku-tuan polinomial terkecil (Alok, 2007). Perhatikan kembali sistem (0.1), transformasi Laplace dari sistem (0.1) diberikan oleh

$$
\begin{aligned}
s \boldsymbol{X}(s)-x(0) & =A \boldsymbol{X}(s)+B \boldsymbol{U}(s) \\
\boldsymbol{Y}(s) & =C \boldsymbol{X}(s) .
\end{aligned}
$$

Karena fungsi transfder didefinisikan untuk syarat awal bernilai nol, maka dapat ditulis

\section{PEMBAHASAN}

\section{Realisasi dari Fungsi Transfer untuk Sistem SISO}

Berikut akan diuraikan proses mendapatkan realisasi dari fungsi transfer suatu sistem SISO. Diberikan suatu fungsi transfer $H(s)$ sebagai berikut

$$
H(s)=\frac{Y(s)}{U(s)},
$$

dimana

$$
Y(s)=b_{n} s^{n}+b_{n-1} s^{n-1}+\cdots+b_{1} s+b_{0}
$$

dan

$$
U(s)=s^{n}+a_{n-1} s^{n-1}+\cdots+a_{1} s+a_{0}
$$

Perhatikan bahwa bentuk (2.1) dapat diubah menjadi

$b_{n}+\frac{\left(b_{n-1}-b_{n} a_{n-1}\right) s^{n-1}+\cdots+\left(b_{1}-a_{1} s\right)+\left(b_{o}-b_{n} a_{0}\right)}{s^{n}+a_{n-1} s^{n-1}+\cdots+a_{1} s+a_{0}}$

sehingga dapat ditulis juga menjadi

$$
Y(s)=b_{n} U(s)+\hat{Y}(s),
$$

dimana

$$
\begin{aligned}
\hat{Y}(s)= & {\left[\left(b_{n-1}-b_{n} a_{n-1}\right) s^{n-1}+\cdots+\left(b_{1}-b_{n} a_{1}\right) s\right.} \\
& \left.+\left(b_{0}-b_{n} a_{0}\right)\right] Q(s)
\end{aligned}
$$

dengan

$$
(s \boldsymbol{I}-A) \boldsymbol{X}(s)=B \boldsymbol{U}(s)
$$

atau dapat juga dituliskan

$$
X(s)=(s I-A)^{-1} U(s)
$$

Dengan $I$ adalah matriks identitias dengan ukuran yang bersesuaian. Akibatnya,

$$
\boldsymbol{Y}(\boldsymbol{s})=\left[\boldsymbol{C}(\boldsymbol{s} \boldsymbol{I}-\boldsymbol{A})^{-1} \boldsymbol{B}\right] \boldsymbol{U}(\boldsymbol{s})
$$

sehingga fungsi transfer untuk sistem adalah

$$
G(s)=C(s I-A)^{-1} B .
$$

$$
Q(s)=\frac{U(s)}{s^{n}+a_{n-1} s^{n-1}+\cdots+a_{1} s+a_{0}} .
$$

Dengan demikian dari (2.3) diperoleh

$$
\begin{gathered}
U(s)=s^{n} Q(s)+a_{n-1} s^{n-1} Q(s)++a_{1} s Q(s) \\
+a_{0} Q(s) \\
s^{n} Q(s)=-a_{n-1} s^{n-1} Q(s)-\cdots-a_{1} s Q(s) \\
-a_{o} Q(s) U(s) .
\end{gathered}
$$

Selanjutnya definisikan

$$
\begin{aligned}
& X_{1(s)}=Q(s) \\
& X_{2(s)}=s Q(s) \\
& X_{3(s)}=s^{2} Q(s) \\
& \quad \vdots \\
& X_{n(s)}=s^{n-1} Q(s)
\end{aligned}
$$

dengan demikian dari (2.3) dan (2.5) menjadi

$$
\begin{aligned}
\hat{Y}(s)= & \left(b_{n-1}-b_{n} a_{n-1}\right) s X_{n-1}(s)+. .+\left(b_{1}-b_{n} a_{1}\right) \\
& s X(s)+\left(b_{0}-b_{n} a_{0}\right) X_{1}(s) \\
s X_{n(s)}= & -a_{n-1} s X_{n-1}(s)-\cdots-a_{1} s X_{1}(s)-a_{0} X_{1}(s) \\
& +U(s) .
\end{aligned}
$$

Sehingga diperoleh

$$
\begin{aligned}
& Y(s)=b_{n} U(s)+\left(b_{n-1}-b_{n} a_{n-1}\right) s X_{n-1}(s)+\cdots \\
& \quad+\left(b_{1}-b_{n} a_{1}\right) s X_{1}(s)+\left(b_{0}-b_{n} a_{0}\right) X_{1}(s) .(2.7)
\end{aligned}
$$


Dengan menggunakan Laplace invers, maka (2.6) dan (2.7) diperoleh

$$
\begin{gathered}
\dot{x}_{n}=-a_{n-1} \dot{x}_{n-1}-\cdots-a_{1} \dot{x}_{1}-a_{0} x_{1}+u, \\
y=b_{n} u+\left(b_{n-1}-b_{n} a_{n-1}\right) \dot{x}_{n-1}+\cdots+ \\
\left.\left(b_{1}-b_{n} a_{1}\right) \dot{x}_{1} b_{0}-b_{n} a_{0}\right) x_{1} .
\end{gathered}
$$

Misalkan

$$
\begin{gathered}
\dot{x}_{1}=x_{2} \\
\dot{x}_{2}=x_{3} \\
\vdots \\
\dot{x}_{n-1}=x_{n}
\end{gathered}
$$

maka (2.10) dan (2.8) secara bersama-sama dapat ditulis menjadi

$$
\dot{\mathbf{x}}(t)=A \mathbf{x}(t)+B \mathbf{u}(t),
$$

dimana

$$
A=\left[\begin{array}{cccc}
0 & 1 & \cdots & 0 \\
0 & 0 & \cdots & 0 \\
\vdots & \vdots & \ddots & \vdots \\
-a_{0} & -a_{1} & \vdots & -a_{n-1}
\end{array}\right]
$$

dan

$$
B=\left[\begin{array}{c}
0 \\
0 \\
\vdots \\
1
\end{array}\right]
$$

Dari (2.9) dan (2.10) diperoleh

$$
\mathbf{y}(t)=C \mathbf{x}(t)+D \mathbf{u}(t)
$$

dimana

$$
C=\left[b_{0}-b_{n} a_{0} b_{1}-b_{n} a_{1} \ldots b_{n-1}-b_{n} a_{n-1}\right]
$$

dan

$$
D=b_{n}
$$

Realisasi dari Fungsi Transfer untuk Sistem MIMO

Berikut akan diuraikan proses mendapatkan realisasi fungsi transfer dari suatu sistem MIMO yaitu memiliki lebih dari satu input dan output. Diberikan suatu fungsi transfer sebagai berikut

$$
H(s)=\left[\begin{array}{ccc}
\frac{N_{11}(s)}{D_{1}(s)} & \cdots & \frac{N_{1 m}(s)}{D_{m}(s)} \\
\cdots & \cdots & \cdots \\
\frac{N_{p 1}(s)}{D_{1}(s)} & \cdots & \frac{N_{p m}(s)}{D_{m}(s)}
\end{array}\right],
$$

Persamaan (2.14) dapat ditulis ulang

$$
H(s)=N(s) D^{-1}(s)
$$

dimana

$$
N(s)=\left[\begin{array}{ccc}
N_{11}(s) & \cdots & N_{1 m}(s) \\
\cdots & \cdots & \cdots \\
N_{p 1}(s) & \cdots & N_{p m}(s)
\end{array}\right],
$$

dan

$$
D(s)=\operatorname{diag}\left[D_{1}(s) D_{2}(s) \cdots D_{m}(s)\right] .
$$

Perhatikan bahwa

$$
\begin{aligned}
& D_{j}(s)=s^{d_{j}}+a_{d_{j}-1}^{j} s^{d_{j}-1}+\cdots+a_{1}^{j} s+a_{0}^{j} \\
& \quad=s^{d_{j}}+L S_{j}(s)
\end{aligned}
$$

dimana

$$
L_{j}=\left[\begin{array}{lll}
a_{0}^{j} a_{1}^{j} & \ldots & a_{d_{j}-1}^{j}
\end{array}\right],
$$

dan

$$
S_{j}(s)=\left[\begin{array}{c}
1 \\
s \\
\vdots \\
s^{d_{j}-1}
\end{array}\right]
$$

untuk $j=1,2, \ldots, m$. Dengan demikian (2.17) menjadi 


$$
\begin{aligned}
D(s) & =\left[\begin{array}{cccc}
s^{d_{1}}+L_{1} S_{1}(s) & 0 & \cdots & 0 \\
0 & s^{d_{2}}+L_{2} S_{2}(s) & \cdots & 0 \\
0 & 0 & \cdots & 0 \\
0 & 0 & 0 & s^{d_{m}}+L_{m} S_{m}(s)
\end{array}\right] \\
& =\left[\begin{array}{cccc}
s^{d_{1}} & 0 & \cdots & 0 \\
0 & s^{d_{2}} & \cdots & 0 \\
0 & 0 & \cdots & 0 \\
0 & 0 & \cdots & s^{d_{m}}
\end{array}\right]+\left[\begin{array}{cccc}
L_{1} & 0 & \cdots & 0 \\
0 & L_{2} & \cdots & 0 \\
0 & 0 & \cdots & 0 \\
0 & 0 & \cdots & L_{m}
\end{array}\right] S(s) .
\end{aligned}
$$

Tulis $\Lambda(s)=\operatorname{diag}\left[s^{d_{1}} s^{d_{2}} \quad \cdots \quad s^{d_{m}}\right]$,

$$
\mathrm{L}=\operatorname{diag}\left[L_{1} L_{2} \quad \cdots \quad L_{m}\right],
$$

dan

$$
\mathrm{S}=\operatorname{diag}\left[S_{1}(s) \quad S_{2}(s) \quad \cdots \quad S_{m}(s)\right],
$$

Maka (2.20) dapat ditulis menjadi

$$
\mathrm{D}(\mathrm{s})=\Lambda(s)+L S(s) .
$$

Dari (2.15) diperoleh

$$
\begin{gathered}
\mathrm{N}(\mathrm{s})=\mathrm{H}(\mathrm{s}) \mathrm{D}(\mathrm{s}) \\
\mathrm{N}_{\mathrm{ij}}(\mathrm{s})=\mathrm{H}_{\mathrm{ij}}(\mathrm{s}) \mathrm{D}_{\mathrm{j}}(\mathrm{s}), \quad \mathrm{i}=1, \cdots, \mathrm{p}, \mathrm{j}=1, \cdots, \mathrm{m}
\end{gathered}
$$

dimana $\mathrm{N}_{\mathrm{ij}}(\mathrm{s})$ adalah elemen dari matriks polinomial $\mathrm{N}(\mathrm{s})$ dan $\mathrm{D}_{\mathrm{j}}(\mathrm{s})$ adalah kelipatan persekutuan terkecil dari semua penyebut pada kolom j. Definisikan

$$
H(\infty)=\lim _{s \rightarrow \infty} H(s),
$$

Maka untuk suatu fungsi transfer skalar $\mathrm{H}_{\mathrm{ij}}(\mathrm{s})$, berlaku

$$
\begin{aligned}
\mathrm{H}_{\mathrm{ij}}(\mathrm{s})-\mathrm{H}_{\mathrm{ij}}(\infty) & =\frac{b_{d_{j}-1}^{j} s^{d_{j}-1}+\cdots+b_{1}^{j} s+b_{0}^{j}}{s^{d_{j}}+a_{d_{j}-1}^{j} s^{d_{j}-1}+\cdots+a_{1}^{j} s+a_{0}^{j}} \\
& =\mathrm{H}_{\mathrm{ij}}(\mathrm{s})
\end{aligned}
$$

dengan realisasi bentuk terkontrol sebagai berikut

dan

$$
\begin{gathered}
A_{j}=\left[\begin{array}{cccc}
0 & 1 & \ldots & 0 \\
0 & 0 & \ldots & 0 \\
\vdots & \vdots & \ddots & \vdots \\
-a_{0}^{j} & -a_{1}^{j} & \vdots & -a_{n-1}^{j}
\end{array}\right], B_{j}=\left[\begin{array}{c}
0 \\
0 \\
\vdots \\
1
\end{array}\right] \\
C_{j}=\left[b_{0}^{j}-b_{d_{j}}^{j} a_{0}^{j} b_{1}^{j}-b_{d_{j}}^{j} a_{1}^{j} \ldots b_{d_{j-1}}^{j}-b_{n}^{j} a_{d_{j-1}}^{j}\right] .
\end{gathered}
$$

Selanjutnya,

$$
\begin{aligned}
\widehat{H}_{i j}(s) D_{j}(s) & =\left[H_{i j}(s)-H_{i j}(\infty)\right] D_{j}(s) \\
& =N_{i j}(s)-H_{i j}(\infty) D_{j}(s) .
\end{aligned}
$$

Misalkan

$$
N_{i j}(s)=b_{d_{j}}^{j} s^{d_{j}}+b_{d_{j}-1}^{j} s^{d_{j}-1}+\cdots+b_{1}^{j} s+b_{0}^{j}
$$

Maka dari (2.24) diperoleh

$$
\begin{aligned}
\widehat{H}_{i j}(s) D_{j}(s) & =b_{d_{j}}^{j} s^{d_{j}}+b_{d_{j}-1}^{j} s^{d_{j}-1}+\cdots+b_{1}^{j} s+b_{0}^{j} \\
& -b_{d_{j}}^{j}\left(s^{d_{j}}+a_{d_{j}-1}^{j} s^{d_{j}-1}+\cdots+a_{1}^{j}+a_{0}^{j}\right) \\
& =\left(b_{d_{j}-1}^{j}-a_{d_{j}-1}^{j} b_{d_{j}}^{j}\right) s^{d_{j}-1}+\cdots+\left(b_{0}^{j}\right. \\
& \left.-a_{0}^{j} b_{d_{j}}^{j}\right)
\end{aligned}
$$

Tulis

$$
C_{j}=\left[\begin{array}{llll}
\beta_{0} & \beta_{1} & \ldots & \beta_{k}
\end{array}\right],(2.24)
$$

dimana

$$
\beta_{k}=b_{k}^{j}-a_{k}^{j} b_{d_{j}}^{j}, \quad k=0,1, \ldots, d_{j}-1 .(2.2
$$

Berdasarkan (2.19) dan (2.24), diperoleh persamaan berikut

$$
\widehat{H}_{i j}(s) D_{j}(s)=C_{j} S_{j}(s) .
$$

Jika $\widehat{H}_{i j}(s)$ adalah elemen dari $\widehat{H}(s)$, maka berdasarkan (2.23) berlaku

$$
\begin{gathered}
\widehat{H}_{i j}(s) D_{j}(s)=C S(s) \\
N(s)-H(\infty) D(s)=C S(s) \\
\quad N(s)=C S(s)+H(\infty) D(s),
\end{gathered}
$$

dimana

$$
C=\left[\begin{array}{llll}
C_{1} & C_{2} & \ldots & C_{m}
\end{array}\right]
$$


sehingga dari (0.3) dan (2.14) diperoleh

$$
\begin{array}{r}
N(s) D^{-1}(s)=C(s I-A)^{-1} B+D \\
{[C S(s)+H(\infty) D(s)] D^{-1}(s)=C(s I-A)^{-1} B+D} \\
C S(s) D^{-1}(s)+H(\infty)=C(s I-A)^{-1} B+D
\end{array}
$$

Akibatnya, diperoleh

$$
D=H(\infty),
$$

dan

$$
\begin{aligned}
S(s) D^{-1}(s) & =(s I-A)^{-1} . \\
B D(s) & =(s I-A) S(s) .
\end{aligned}
$$

Perhatikan bahwa

$$
\begin{aligned}
& B_{j} D_{j}(s)=\left[\begin{array}{c}
0 \\
0 \\
\vdots \\
1
\end{array}\right]\left(s^{d_{j}}+a_{d_{j}-1}^{j} s^{d_{j}-1}+\ldots+a_{1}^{j} s+a_{j}^{0}\right. \\
& =\left[\begin{array}{c}
0 \\
0 \\
\vdots \\
s^{d_{j}}+a_{d_{j}-1}^{j} s^{d_{j}-1}+\ldots+a_{1}^{j} s+a_{j}^{0}
\end{array}\right] \\
& =\left[\begin{array}{ccccc}
\mathrm{s} & -1 & 0 & \cdots & 0 \\
0 & s & -1 & \cdots & 0 \\
\vdots & \ddots & \ddots & \ddots & \vdots \\
a_{0}^{j} & a_{1}^{j} & a_{2}^{j} & \cdots & s+a_{d_{j}-1}^{j}
\end{array}\right]\left[\begin{array}{c}
1 \\
s \\
\vdots \\
\mathrm{s}^{\mathrm{d}_{j}-1}
\end{array}\right] \\
& =\left(s I-A_{j}\right) S_{j}(s) \text {. }
\end{aligned}
$$

Akibatnya, dari (2.31) diperoleh

\section{KESIMPULAN}

Misalkan $H(s)$ adalah suatu fungsi transfer sedemikan sehingga

$$
H(s)=C(s I-A)^{-1} B+D,
$$

Jika $H(s)$ berbentuk fungsi skalar maka realisasi dari $H(s)$ adalah

$$
A=\operatorname{diag}\left[\begin{array}{lll}
A_{1} & A_{2} & \ldots A_{m}
\end{array}\right] .
$$

dimana

$$
A=\left[\begin{array}{cccc}
0 & 1 & \cdots & 0 \\
0 & 0 & \ddots & 0 \\
\vdots & \vdots & \ddots & 1 \\
-a_{0}^{j} & -a_{1}^{j} & \cdots & -a_{d_{j}-1}^{j}
\end{array}\right]
$$

dan

$$
B=\operatorname{diag}\left[\begin{array}{llll}
B_{1} & B_{2} & \ldots & B_{m}
\end{array}\right]
$$

dengan

$$
\mathrm{B}_{\mathrm{j}}=\left[\begin{array}{c}
0 \\
0 \\
\vdots \\
1
\end{array}\right]
$$




$$
B=\left[\begin{array}{c}
0 \\
0 \\
\vdots \\
1
\end{array}\right]
$$

$C=\left[b_{0}-b_{n} a_{0} b_{1}-b_{n} a_{1} \ldots b_{n-1}-b_{n} a_{n-1}\right]$

dan

$$
D=b_{n} .
$$

Jika $H(s)$ berbentuk suatu matriks, maka realisasi dari $H(s)$ adalah

\section{DAFTAR PUSTAKA}

Alok, S. 2007. Linear Systems Optimal and Robust Control. Francis: CRC Press.

Antsaklis, P. J dan Anthony N, Michel. 2007. A Linear Systems Primer. Boston: Birkhauser.

Hendricks E, Jannerup, O dan Sorensen, P. H. 2008. Linear System Control. Springer, Heidelberg.

Kaczorek, T. 1992. Linear Control System. Galliard: Great Yarmouth.

Ogata, K. 2002. Modern Control Engineering. New Jersey:

Prentice-Hall.

$$
\begin{aligned}
B & =\operatorname{diag}\left[\begin{array}{llll}
B_{1} & B_{2} & \ldots & B_{m}
\end{array}\right], \\
C & =\left[\begin{array}{llll}
C_{1} & C_{2} & \ldots & C_{m}
\end{array}\right], \\
D & =H(\infty)=\lim _{s \rightarrow \infty} H(s) .
\end{aligned}
$$

dimana masing-masing $A_{j}$ berbentuk seperti (3.2), masing-masing $B_{j}$ berbentuk seperti (3.3), dan masing-masing $C_{j}$ berbentuk seperti (3.4). 